\title{
Research Paper \\ Evaluation of Appropriate Metatarsal Pad Length on Mechanical Parameters of Diabetic Foot Ulcer During Walking
}

\author{
Ebrahim Abdi ${ }^{1}\left(\mathbb{D}\right.$, Mansour Islami ${ }^{2},{ }^{*}$ Mohammad Taghipour $^{3}{ }^{\circ}$, Ali Bakhtiari ${ }^{4}$
}

1. Department of Sport Biomechanics, Education Center and Faculty of Sport Sciences, Kavoshgaran Danesh and Pouyesh Vieira, Tehran, Iran.

2. Department of Sports Biomechanics, Faculty of Physical Education and Sports Sciences, University of Mazandaran, Mazandaran, Iran.

3. Department of Physiotherapy, Mobility Impairment Research Center, Babol University of Medical Sciences, Mazandaran, Iran

4. Department of Sport Physiology, Faculty of Physical Education and Sports Sciences, University of Tehran, Tehran, Iran.

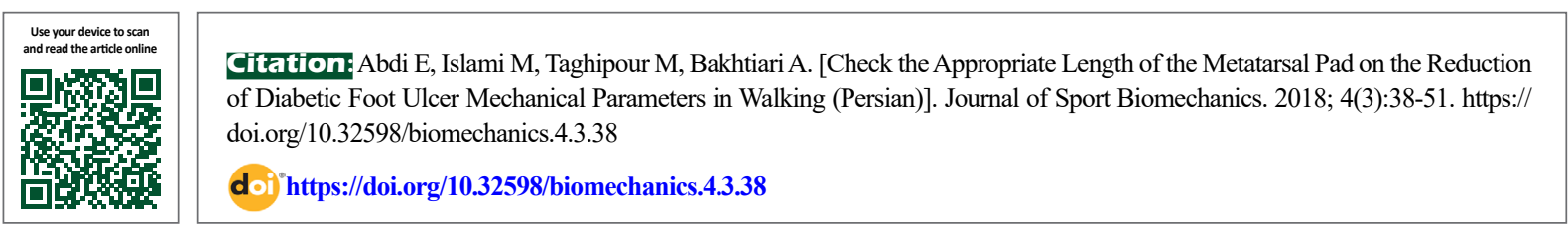

\section{(i)}

Article Info:

Received: 27 Aug 2018

Accepted: 03 Nov 2019

Available Online: 01 Dec 2018

Keywords:

Diabetics foot ulcer,

Peak plantar pressure,

Time-pressure inte-

gral, Metatarsal pad

\section{AB STRACT}

Objective: Studies have reported that the best strategy for reducing the peak pressure on metatarsals is to increase the surface area with the metatarsal pad. but the optimal length of metatarsal pads is still unclear. The purpose of this study was to evaluate the appropriate length of metatarsal pad and its effect on the reduction of diabetic foot ulcer mechanical parameters while walking

Methods: Participants were 15 male patients with diabetes mellitus (Mean $\pm S D=63.3 \pm 2.01$ years). Handmade metatarsal pads with a length equal to 18,20, 23 and $25 \%$ of foot length were used. Patients wearing these pads passed the RS-scan foot scan in 5 separate conditions and their data were recorded. Repeated measures Analysis of Variance (ANOVA) was used for analyzing data.

Results: Peak pressure significantly decreased in metatarsal regions 1,3 and $4(P=0.001)$, but no significant difference was observed in regions 2 and $5(P<0.05)$. A significant difference was observed in pressure-time integral between the five conditions in all metatarsal pads $(P=0.001)$, but its change between the type of pad and metatarsal region was different. In the metatarsal regions 1 and 2 with pads of 18 and $20 \%$ of foot length, the peak time pressure was significantly reduced, while in the pads of 23 and $25 \%$ of foot length, it significantly increased. In the metatarsal regions 3, 4 and 5 with a pad of $18 \%$ of foot length, the peak time pressure increased, while with the pads of 20,23 , and 25 of foot length, a reduction was observed.

Conclusion: As the pad length increases, the pressure peak in the metatarsal areas decreases, but the effect of pad length on the time pressure depends on the metatarsal region and the pad length. The length of the pad relative to the size of the foot is a step forward for the insole design. Insole designers are recommended to use a metatarsal pad with $23 \%$ of foot length for diabetic patients.

\section{Extended Abstract \\ tion's report in 2003, 194 million people were estimated to \\ 1. Introduction

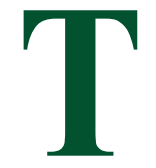 \\ he worldwide prevalence of diabetes has dramatically increased, and $10 \%$ of the Iranian population has this disease [1]. According to the World Health Organiza- \\ have diabetes worldwide, predicted to reach 366 million by 2030. In the 2010 census, this rate increased to 374 million worldwide $[1,2]$. The major problems in diabetic patients consist of foot infection and ulcer, ultimately leading to amputation [4]. Studies suggested that $20 \%$ of hospitalized Iranian diabetic patients experience foot problems [3]. \\ * Corresponding Author: \\ Mohammad Taghipour, PhD. \\ Address: Department of Physiotherapy, Mobility Impairment Research Center, Babol University of Medical Sciences, Mazandaran, Iran. \\ Tel: +98 (912) 6899352 \\ E-mail: taghipourm@yahoo.com}


Increased local pressure and the pressure-time on the sole are the most critical mechanisms of the mechanical risk of plantar foot ulcer in diabetic patients $[5,6]$. In diabetic patients, due to the limited mobility, the shock absorption mechanism of the wrist and knee joints is reduced [7]. A study indicated that the most prevalent foot ulcers are in the metatarsal areas of diabetic patients [18]. The peak of pressure and the time of pressure are highly correlated with the ulceration of the foot; thus, the best strategy to reduce the peak pressure in metatarsal areas is to increase the contact area. In this regard, the metatarsal pad is used to increase the contact surface and reduce the pressure peak [22]. This study aimed to determine the proper metatarsal pad length for reducing foot plantar pressure parameters (peak pressure and pressure time). As a result, we measured the ratio of the metatarsal pad length to the actual foot length.

\section{Patients and Methods}

In total, 15 male patients with type 2 diabetes (Mean age: 63.3 y) were selected among 2000 diabetic patients in Babolsar County, Iran. They were selected by assessing their medical records and using a convenience sampling technique. A foot scan system (RS-scan, Belgium) with $1 \times 0.4$ $\mathrm{m}$ dimensions at $50 \mathrm{fps}(\mathrm{Hz})$ was used for measuring the foot pressure and pressure distribution pattern. Handmade metatarsal pads with lengths equal to $18 \%, 20 \%, 23 \%$, and $25 \%$ of foot length were used. Figure 1 shows the RS-scan system and the used pads. The study variables were the peak plantar pressure and the pressure-time on 10 plantar regions (heel, center, metatarsal, \& thumb). Among these regions, the peak plantar pressure and the pressure-time on 5 metatarsal regions were measured and used for the statistical analysis. The anthropometric characteristics of the subjects' feet were measured after explaining the study and test methods to them and obtaining informed consent from them. A 12-meter straight path was determined and the foot scan system was longitudinally placed at a distance of 10 $\mathrm{m}$. The study subjects were requested to start walking with the optional and preferable speed from the beginning of the route and maintain their speed until its end. We adjusted the patients' walking speed based on the forms of the gait cycle's supporting phase in the scanning system; the study subjects' walking speed did not vary during the test.

\section{Results}

According to Figure 1 and the one-way repeated measures Analysis of Variance (ANOVA) results, the peak pressure increased with enhancing pad length in the metatarsal region 1; the within-group difference between pads was significant $(\mathrm{P}=0.001)$. The descriptive indices also revealed a decrease of $24 \%$ in the $18 \%$ pad. Furthermore, in the pads of $20 \%, 23 \%$, and $25 \%$ of foot lengths, there were $36 \%$, $40 \%$, and $43 \%$ decreases in the peak pressure parameter, respectively. Moreover, ANOVA results of pressure time on the first metatarsal head section suggested a significant difference in the peak time of pressure between feet with and without pad conditions $(\mathrm{P}=0.001)$.

\section{Discussion}

The present study investigated the appropriate length of the metatarsal pad and its effect on the mechanical parameters of diabetic foot ulcer during gait. According to the obtained results, the distribution pattern of plantar pressure and the time of applying pressure on the metatarsals changed with the application of pads. In other words, the distribution of plantar pressure depends on the distribution of force and the surface at which the force is applied; thus,

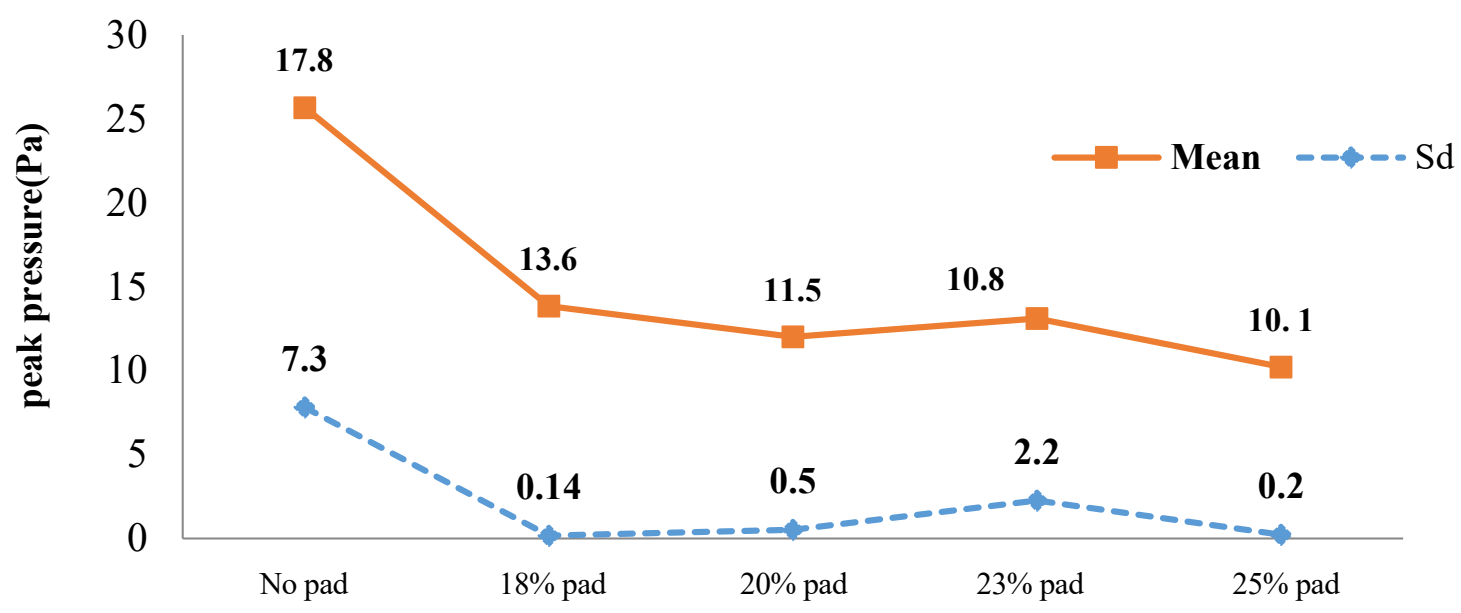

Figure 1. Descriptive statistics (Mean $\pm \mathrm{SD}$ ) and peak pressure under 5 different conditions

*Significant difference between pads in the metatarsal region 1 
by changing the surface, the distribution of plantar pressure changes. Therefore, when the pads' length percentages increase, the peak pressure level decreases. This is because using a metatarsal pad increases the contact level in the metatarsal region. Reducing peak pressure in metatarsal regions is a crucial advantage of pad use.

\section{Conclusion}

The greatest effect of metatarsal pads was detected in metatarsal regions 1,3, and 4. As the pad length increased, the pressure peak in the metatarsal areas decreased. The highest pressure reduction was observed in the metatarsal head with pads of $23 \%$ and $25 \%$ of foot length. Since the pad length increased in the metatarsal regions 1 and 2 with increasing pad length, the $23 \%$ of foot length could be an appropriate pad length to reduce the peak pressure and the time of pressure in metatarsal areas. Insole designers are recommended to use a metatarsal pad with $23 \%$ of foot length for diabetic patients

\section{Ethical Considerations}

Compliance with ethical guidelines

All ethical principles were considered in this article. The participants were informed about the purpose of the research and its implementation stages; they were also assured about the confidentiality of their information; Moreover, They were allowed to leave the study whenever they wish, and if desired, the results of the research would be available to them.

Funding

This research did not receive any specific grant from funding agencies in the public, commercial, or not-forprofit sectors.

\section{Authors' contributions}

All authors contributed equally in preparing all parts of the research.

\section{Conflicts of interest}

The authors declared no conflict of interest.

\section{Acknowledgements}

The authors would like to thank the staff of Biomechanics Laboratory at Faculty of Physical Education and Sports Science, University of Mazandaran. 


\title{
بررسى طول مناسب يد متاتارس روى كاهش يار امترهاى مكانيكى زخم پايى ديابتى در حين راهرفتن
}

\author{
ابراهيم عبدى' ن، منصور اسلامى'، "محمد تقىيور' ه، على بختيارى"

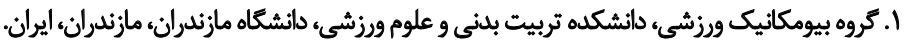

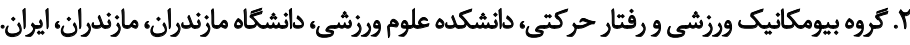

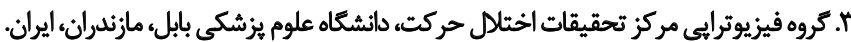

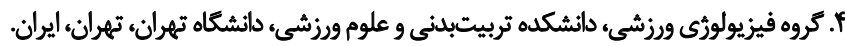

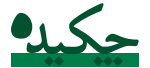

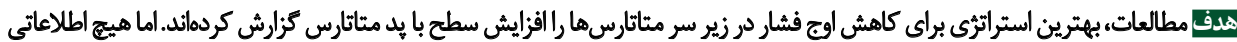

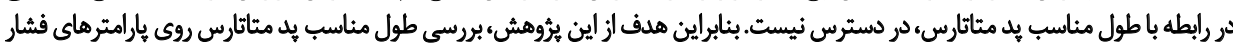

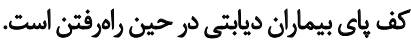

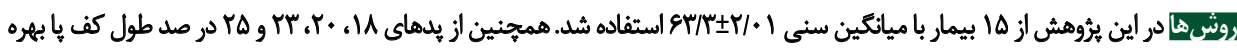

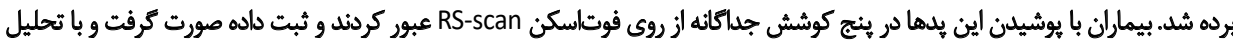

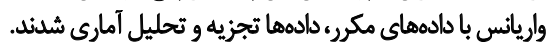

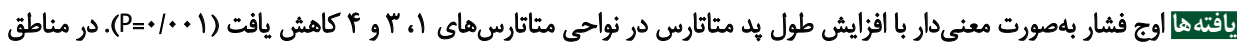

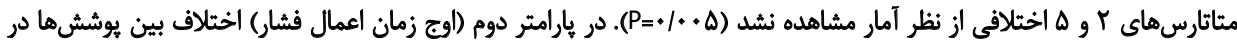

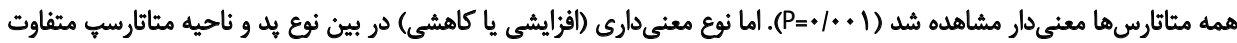

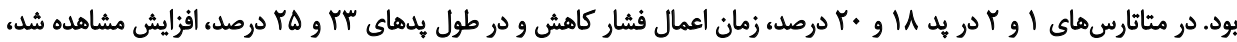

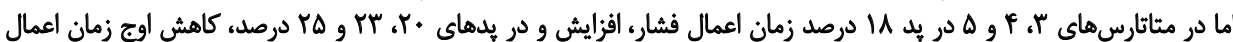
فشار مشاهلده شد.

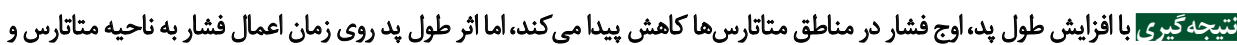

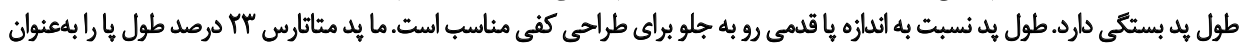

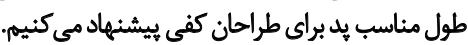

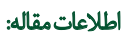

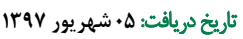

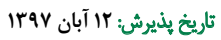

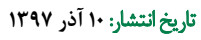

كليدواروها:

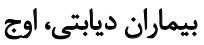
فشار، زمان اعمال فشار و و يد مثياتارس

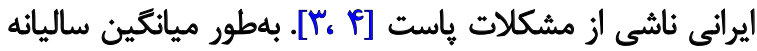

مقدمه

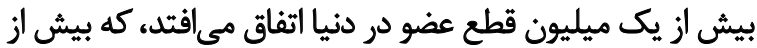

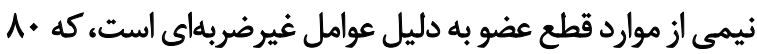

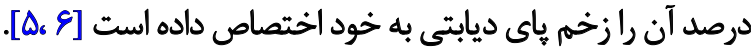

افزايش موضعى فشار و زمان اعمال فشار در كف با، مهمثرين

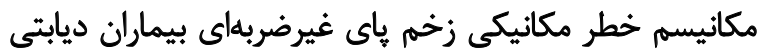

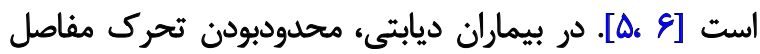

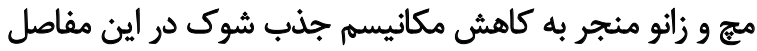

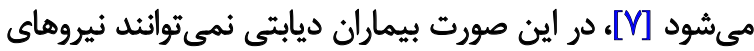

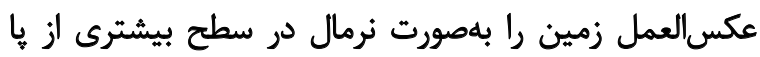

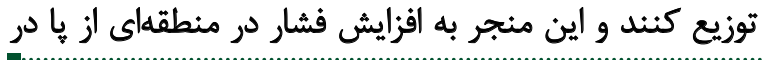

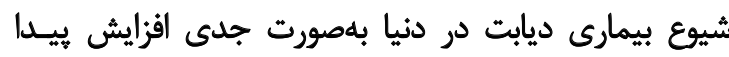

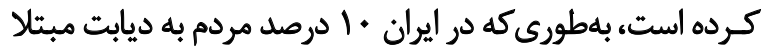

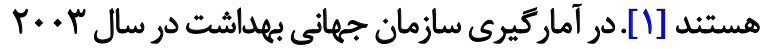

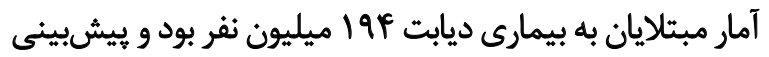

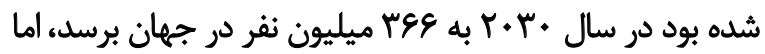

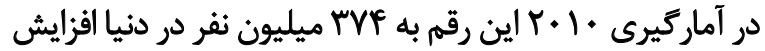

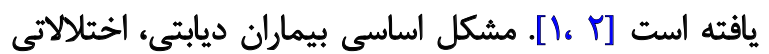

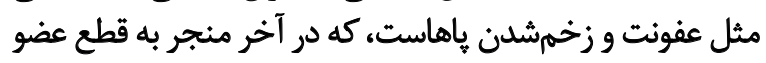

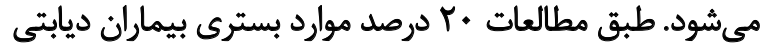

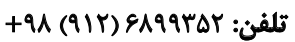
taghipourm@yahoo.com : بلفت الكثرونيكي 
بين سر متاتارسها با زمين افزايش ييدا كند، كه درنهايت منجر

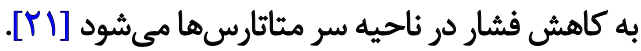
با توجه به مطالعات يادشده، استفاده از بِد متاثارس منجر

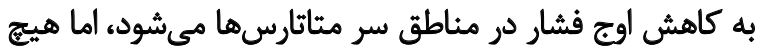

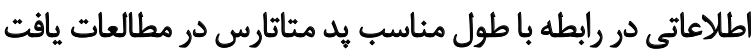

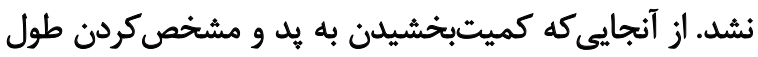

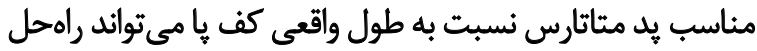

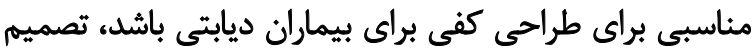

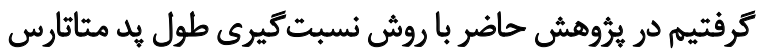

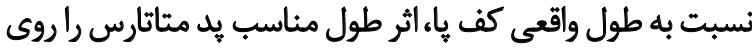

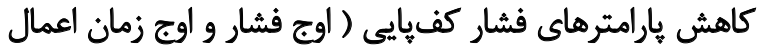

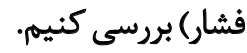

$$
\text { ووش شُشاسي }
$$

در يروهش حاضر، ها نفر مرد مبتلا به ديابت نوع دوم با باري

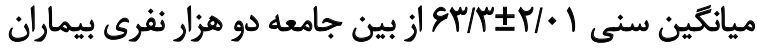

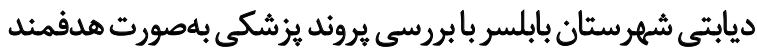

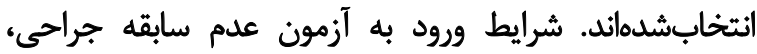

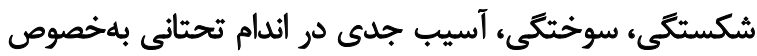

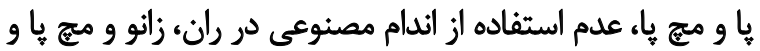

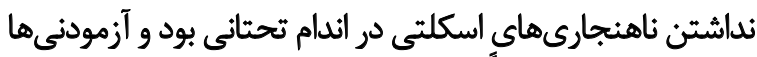

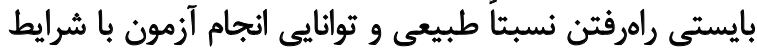

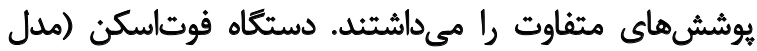

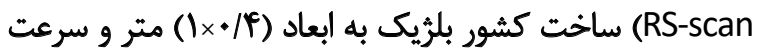

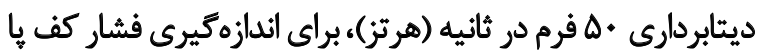

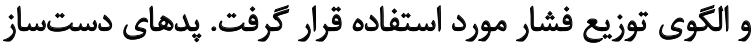

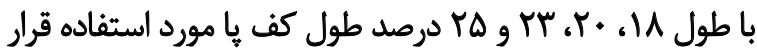

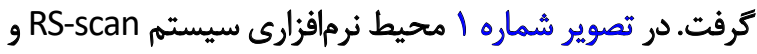
انواع بدها را نشان داده شده است.

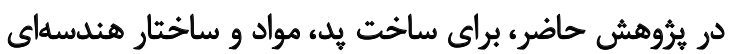

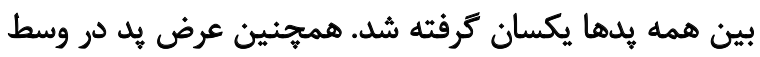

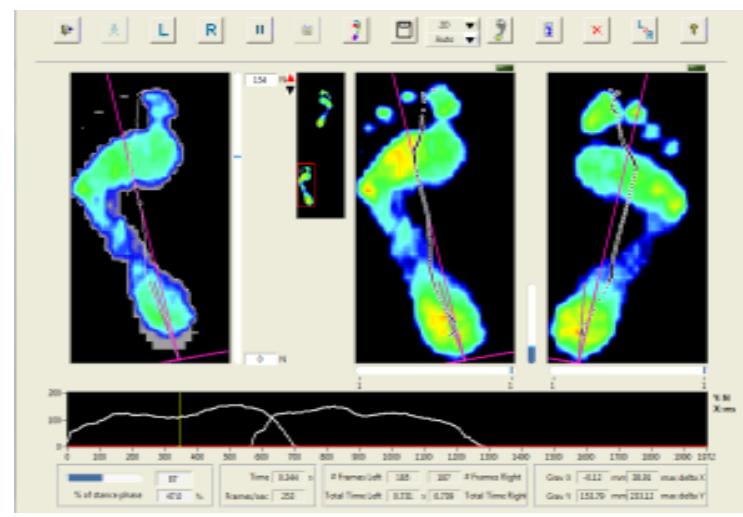

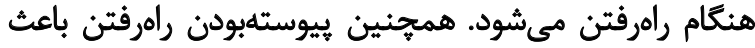

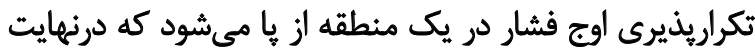

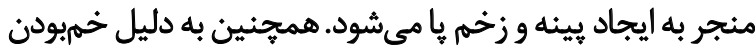

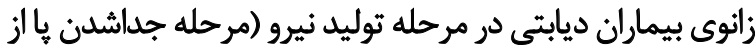

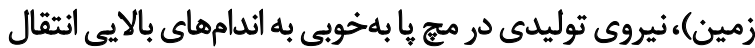

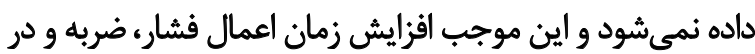

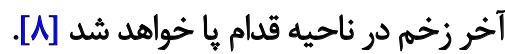

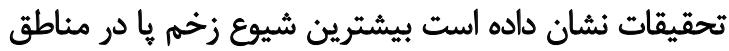

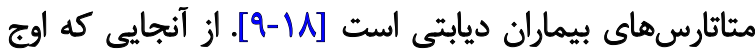

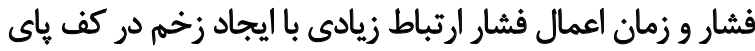

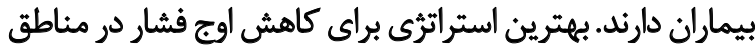

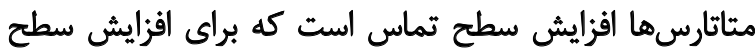

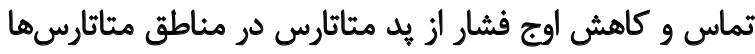

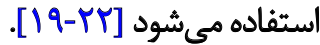

تحقيقات نشان داده است كه بد مثاتارس به علت اينكه فشار

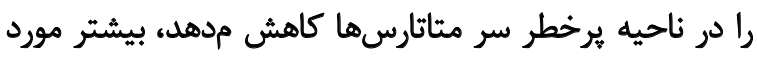

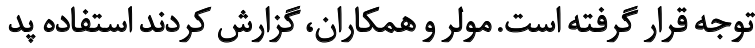

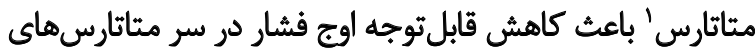

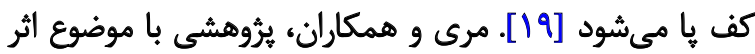

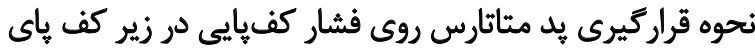

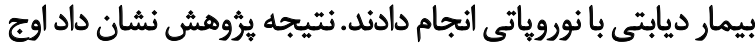

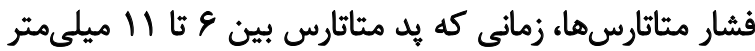

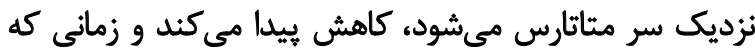

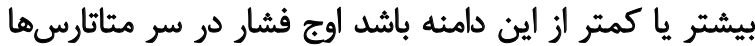

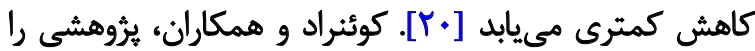

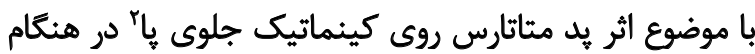

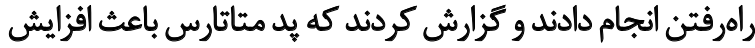

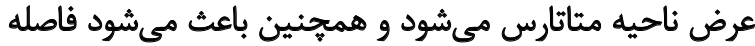

\section{Metatarsal pad \\ 2. Fore foot}

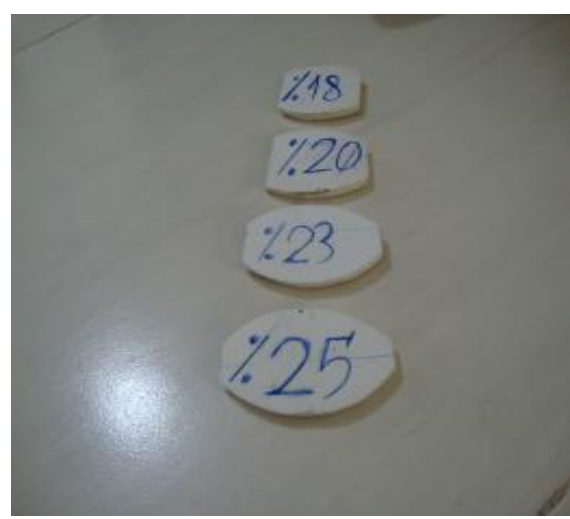

تصوير ا. محيط نرمافزار كيت فوتاسكن و يدها 
جدول ا. طول يد به درصد طول كف يا و به سانتىمتر

\begin{tabular}{|c|c|c|}
\hline شماره كفش & ميانكين طول كف يا بين + ا نقر به سانتىمتر تبديل به درصد & تبديل درصد متغير طول بد مثاتارس به سانتىمتر \\
\hline \multirow{4}{*}{ fi } & \multirow{4}{*}{$M f / \Delta=1 \ldots$} & $r e(\Delta x) \Delta=r / P 1$ \\
\hline & & $r \Gamma / \Delta \times Y=r / Q$ \\
\hline & & 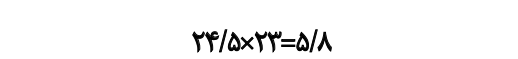 \\
\hline & & $r \varphi / \Delta \times r \Delta=F / I$ \\
\hline \multirow{4}{*}{ fr } & \multirow{4}{*}{$r=1 .}$. & $r e \times \mid \Lambda=r / 8$ \\
\hline & & $r E \times Y=\Delta / T$ \\
\hline & & $r \varepsilon \times M r=\Delta / Q A$ \\
\hline & & 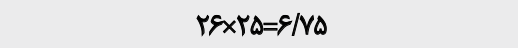 \\
\hline \multirow{4}{*}{$p r$} & \multirow{4}{*}{$T V=1 \ldots$} & $r V \times M=F / N$ \\
\hline & & $M X X+=\Delta / T^{e}$ \\
\hline & & $T V X Y M=E / M I$ \\
\hline & & $T V \times Y \Delta=V / T q$ \\
\hline
\end{tabular}

مجله بيومكانيك وزنث

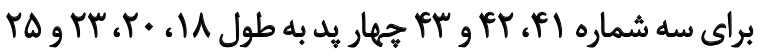

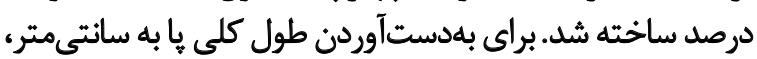

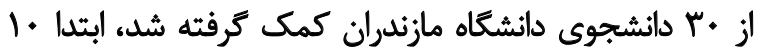

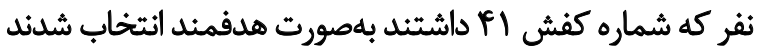

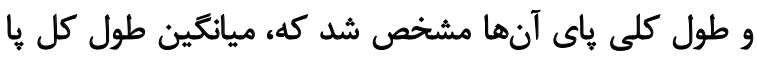

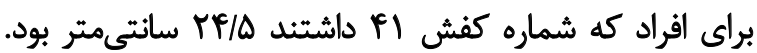

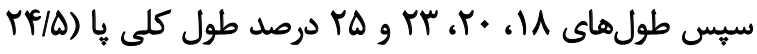

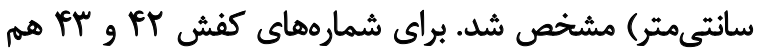

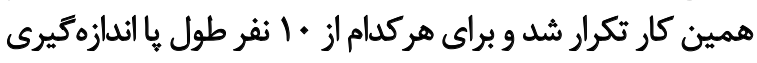

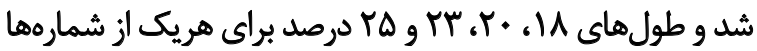

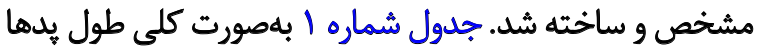

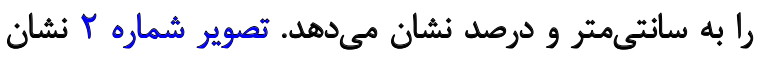

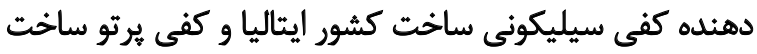

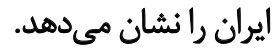

$(+$

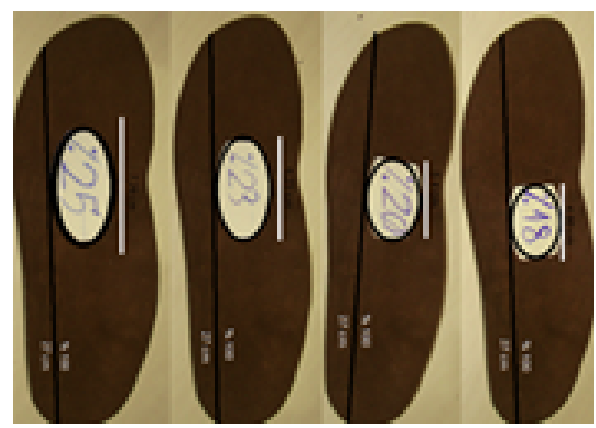

مجله بيومكانيك ولنش
جهار سانتى متر و به طرف سر متاثارس ها دو سانتى متر و بهطرف

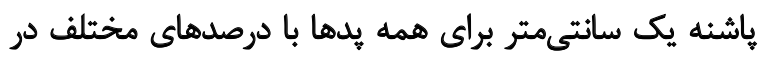

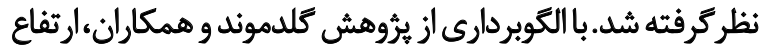

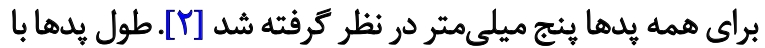

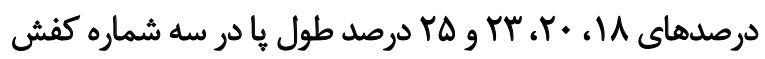

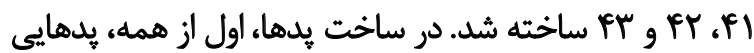

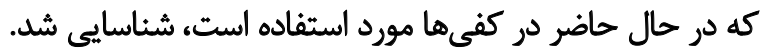
بهاطورى كه در كفي سيليكوني ساخت كشيور ايتاليا براي شيماره

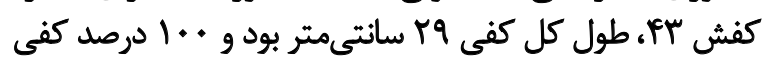
راشامل مى شد و طول بد مثاتارس در كفى شش سائتى متر بود،

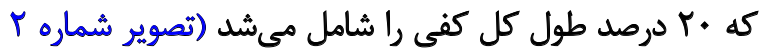

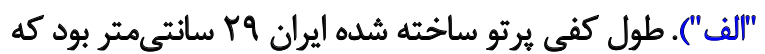

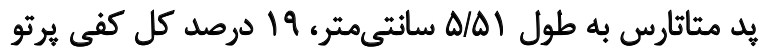

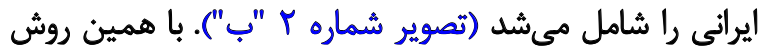

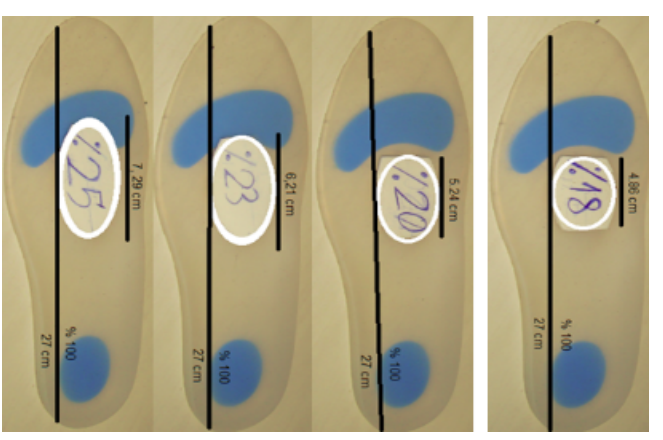

تصوير r. الف) كفى سيليكونى ساخت ايتاليا، ب) كفى يرثو ساخت ايران 
سيستم فوت اسكن، سرعت راهرفتن بيمار را تنظيم ميش وشد. كل

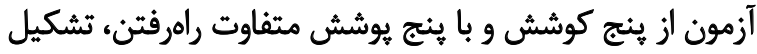

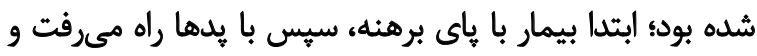

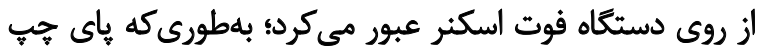

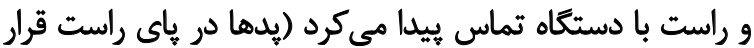

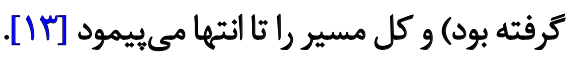

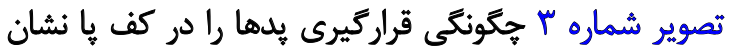

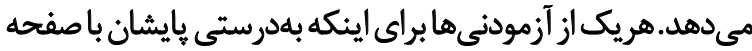

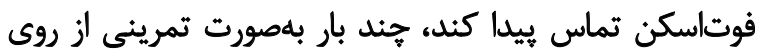

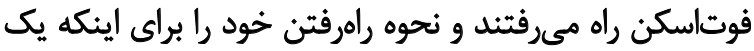

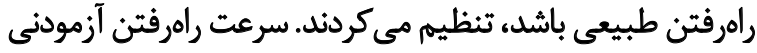

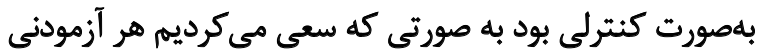

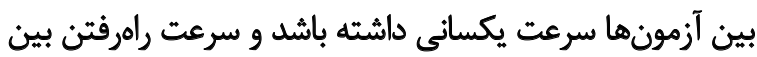

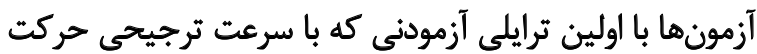

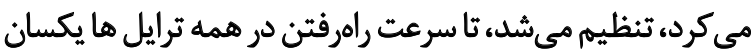

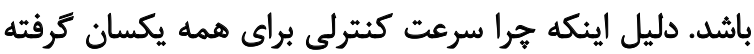

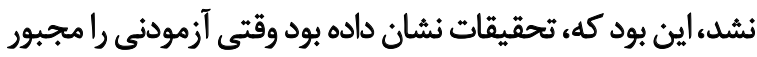

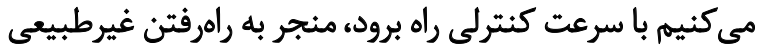

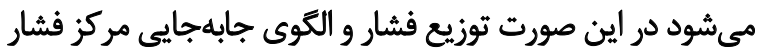

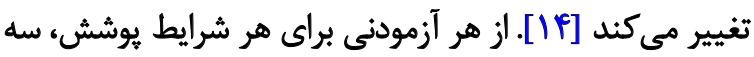

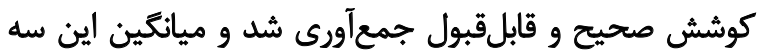
كوشش بهعنوان اطلاعات مربوط به هر آزمودنى ثبت شيت شد. از نسخه • ب نرمافزار SPSS جهت بروسى آمارى استفاده شد.

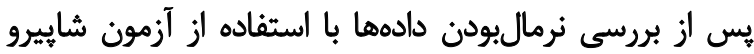

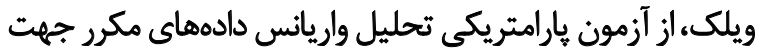

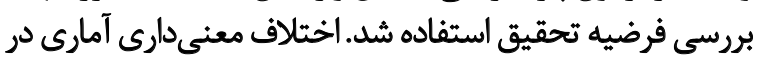

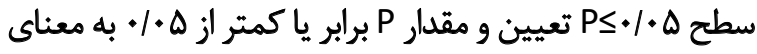
رد فرض صفر در نظر كرفته شد.

ثتايج با توجه به نتايج كزارش شده در تصوير شماره f و آزمون تحليل

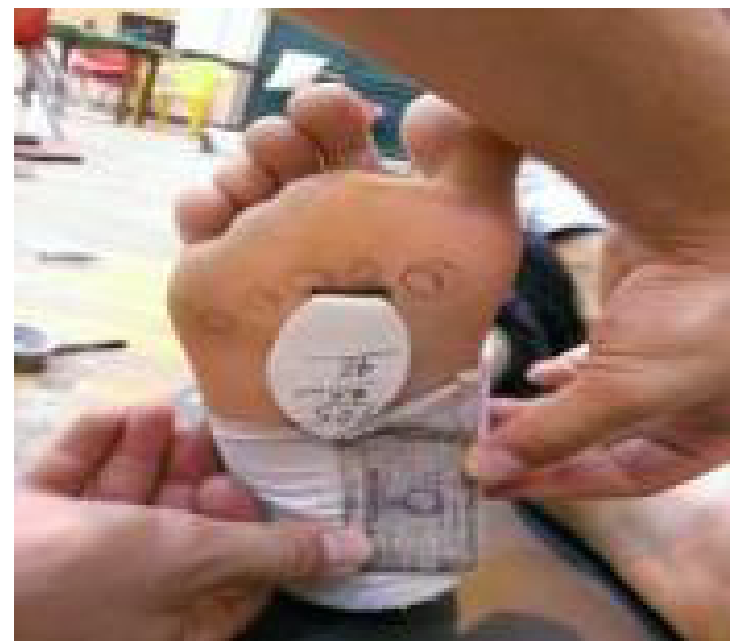

مجله بيومكانيك ولشث تصوير ז. نحوه قراركيرى بد در كف ها

متغيرهاى آزمون شامل حداكثر فشار كفياييى و اوج زمان

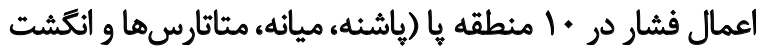

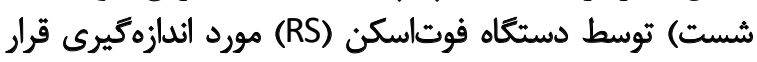

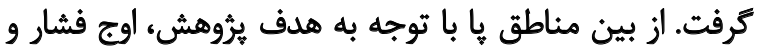

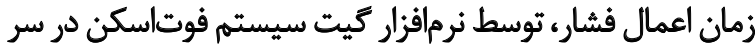
ينج متاتارس كف با اندازهيرى و براى تحليل آمارى آماده شد. اين يُؤهش در آزمايشعاه تحقيقاتى بيومكانيك ورزشى دانشعاه

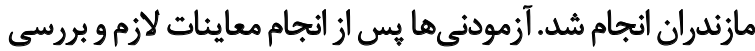

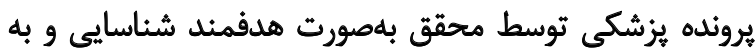

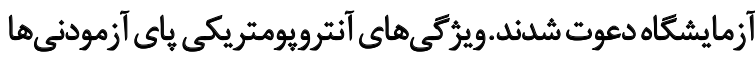

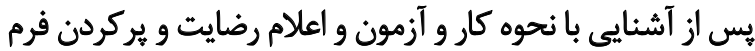

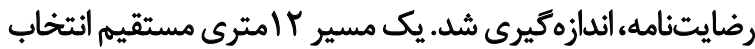

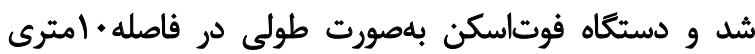

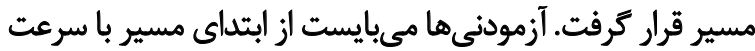

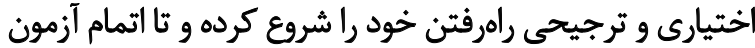

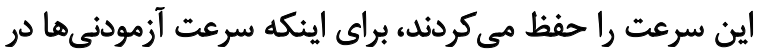

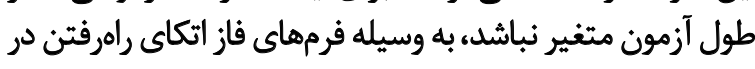

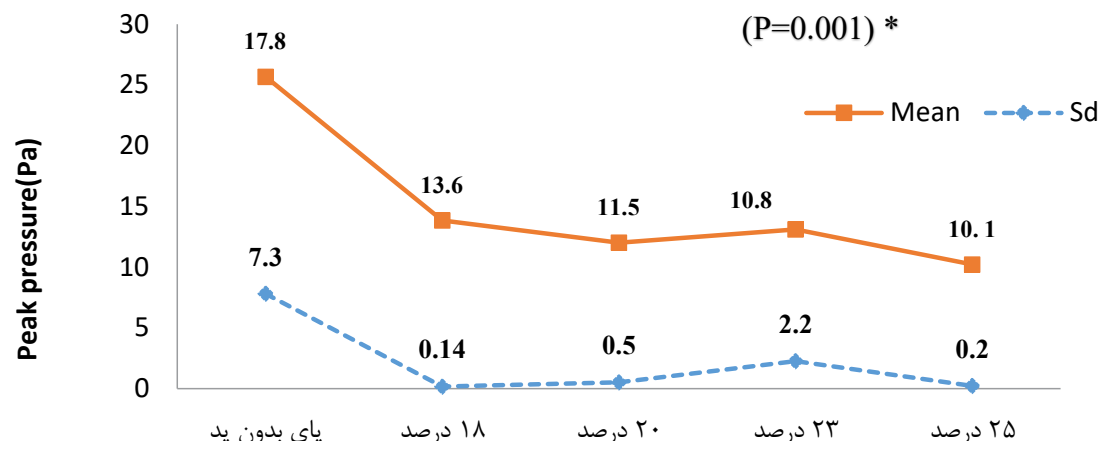

مجله بيومكانيك ولانش

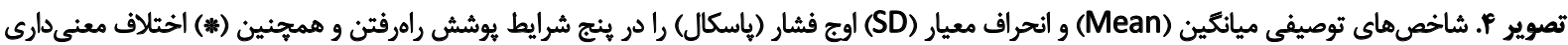

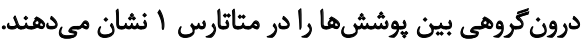




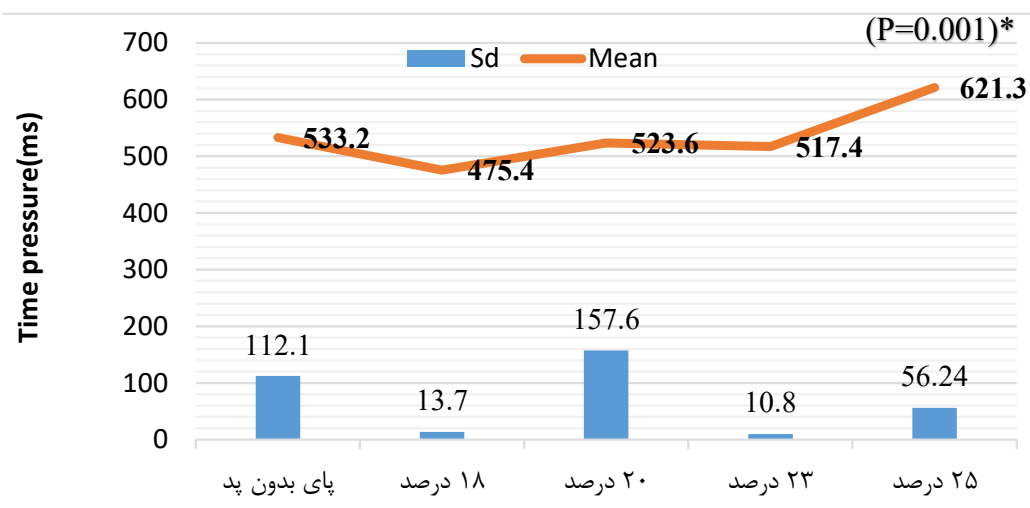

مجله بيومكانيك ورنش (هن

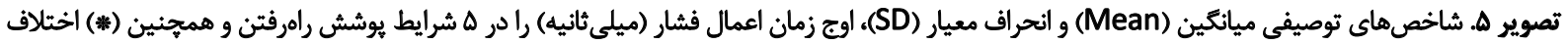

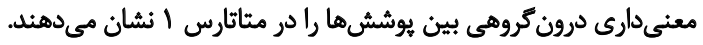

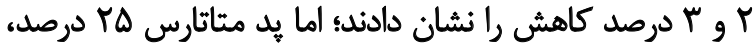

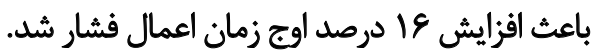

نتايج كزارش شده در تصوير شماره ع و آزمون تحليل واريانس

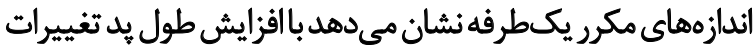

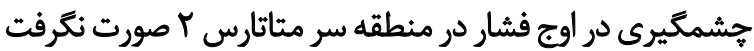

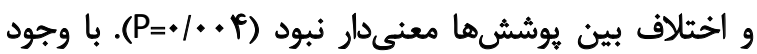

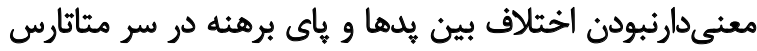

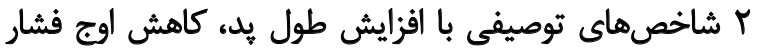

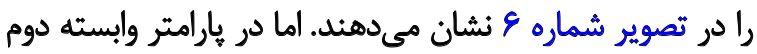

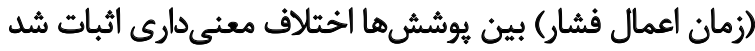

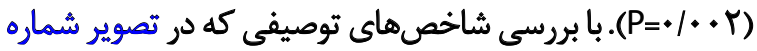

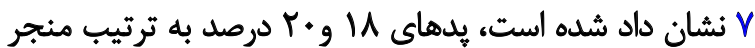

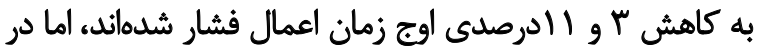

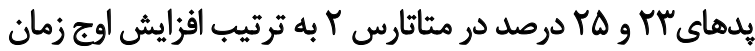

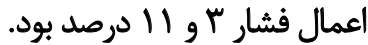

با توجه نتايج كزارششده در تصوير شماره \ه، با افزايش طول

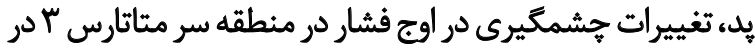

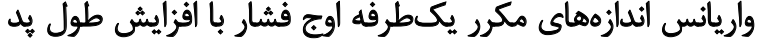

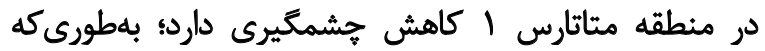

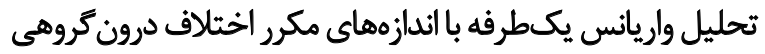

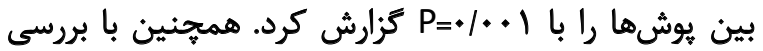

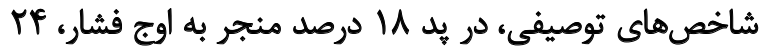

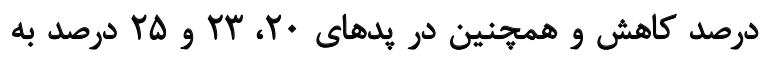

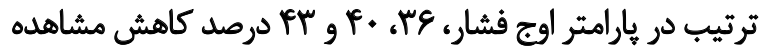

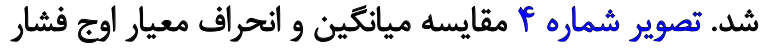

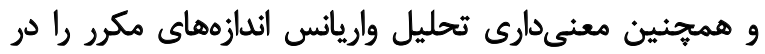
كوشش هاى مختلف در منطقه سر مثاتارسال النشان مي مدهدي. بررسى زمان اعمال فشار (يارامتر وابسته دوم) در سر متاثارس

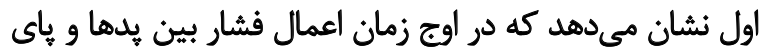

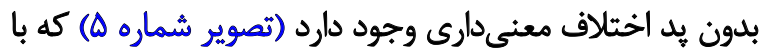

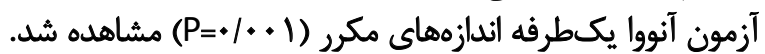

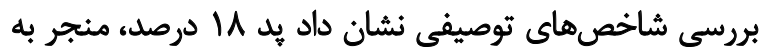

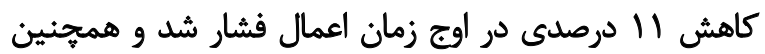

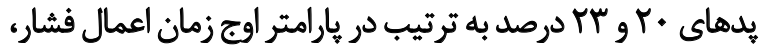

مجله بيومكانيك وزنش

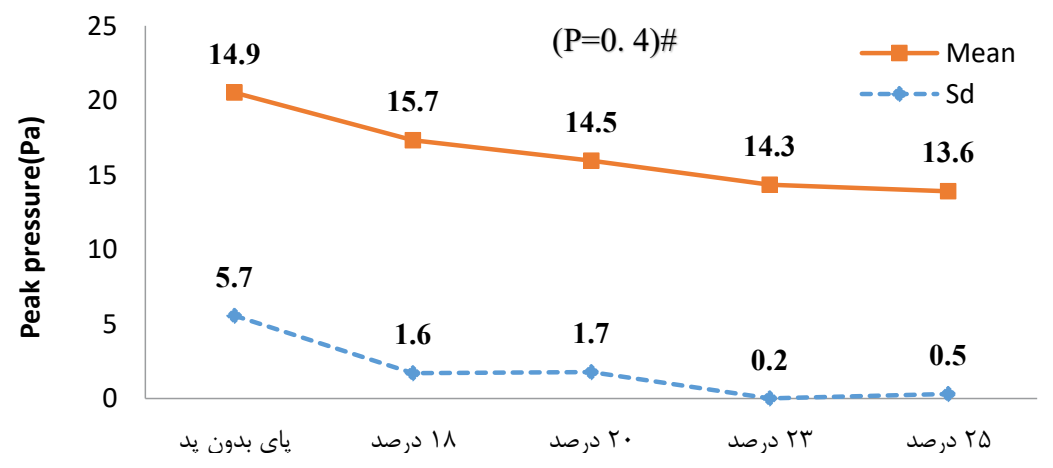

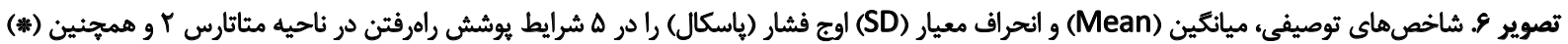

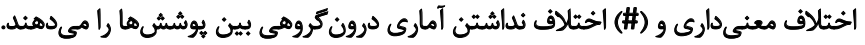




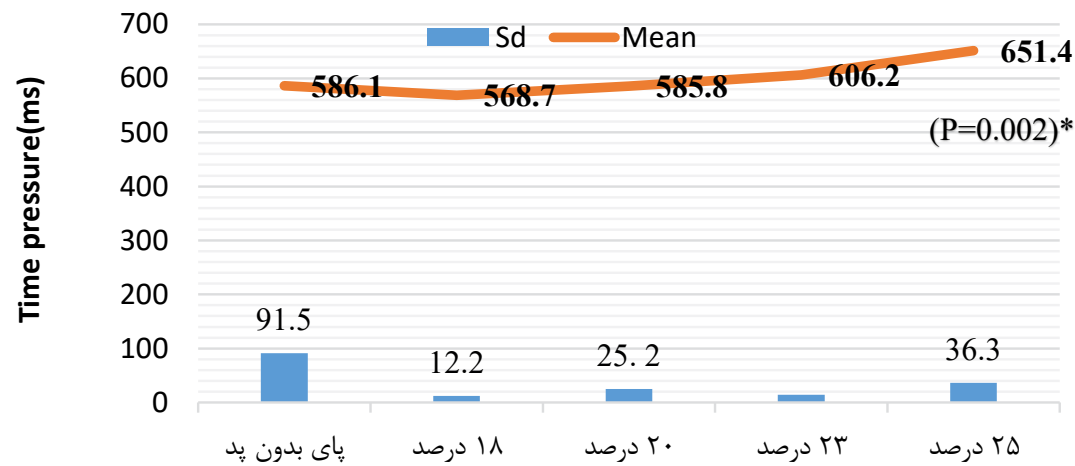

مجلله بيومكانيك ورنش

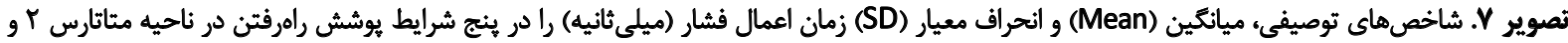

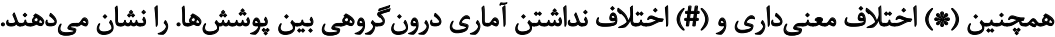

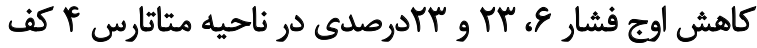

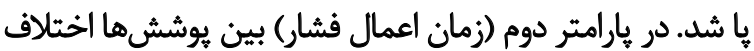

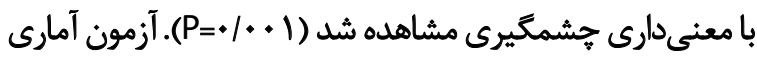

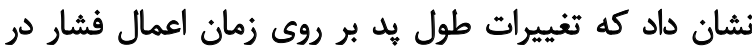

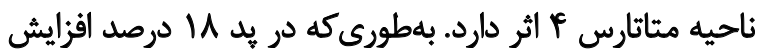

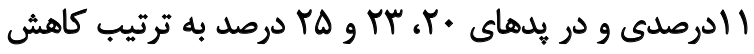

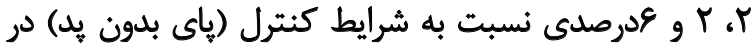

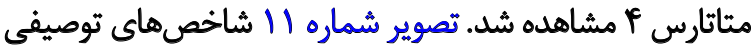
زمان اعمال فشار را نشان مى مهد. تصويد.

با توجه به نتايج كزارش شده در تصوير شمار مار با با افزايش

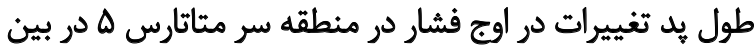

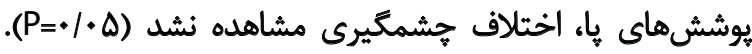

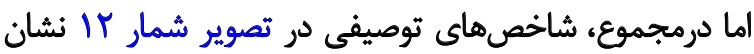

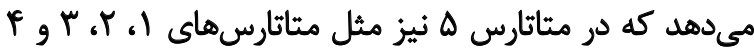

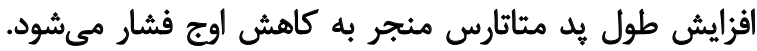

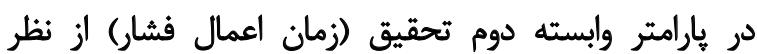

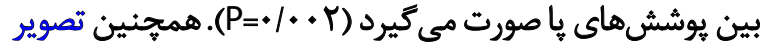

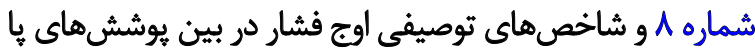

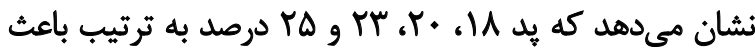

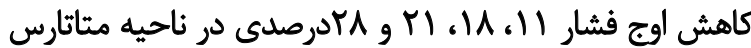

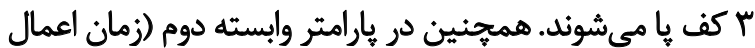

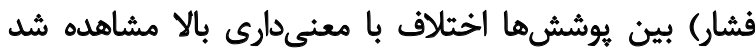

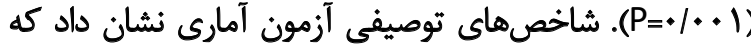

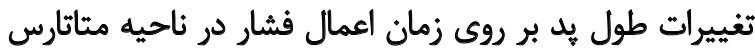

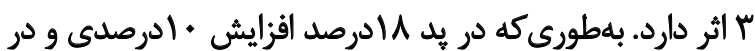

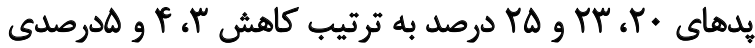

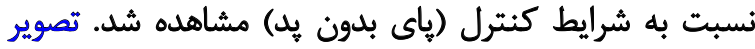

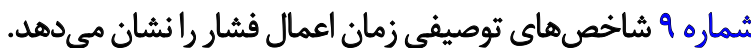

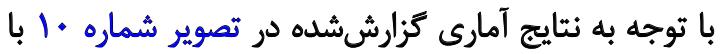

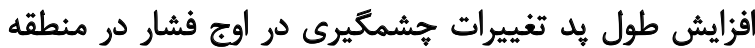

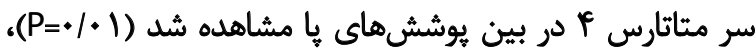

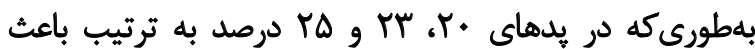

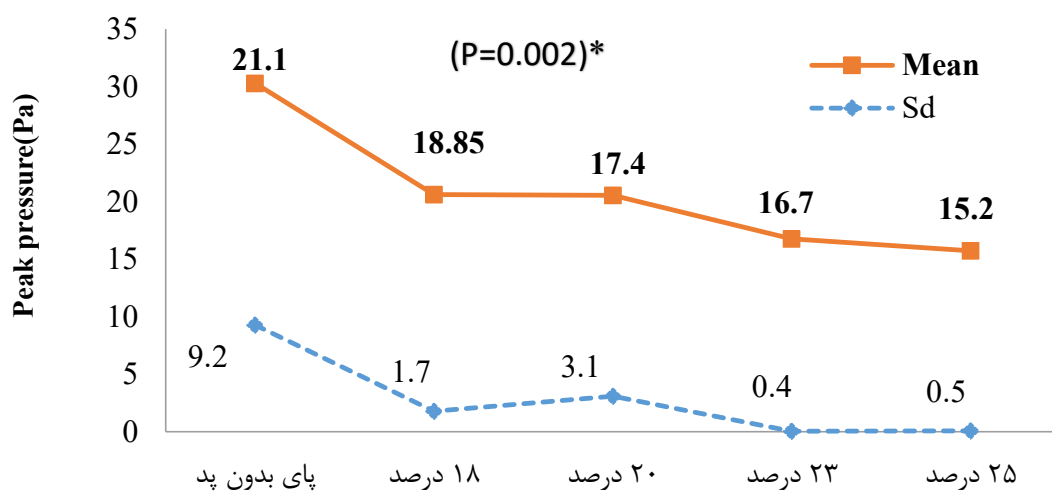

مجله بيومكانيك ولنش

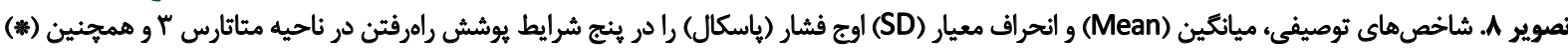

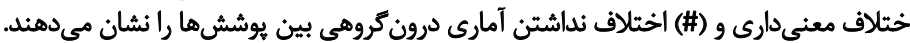




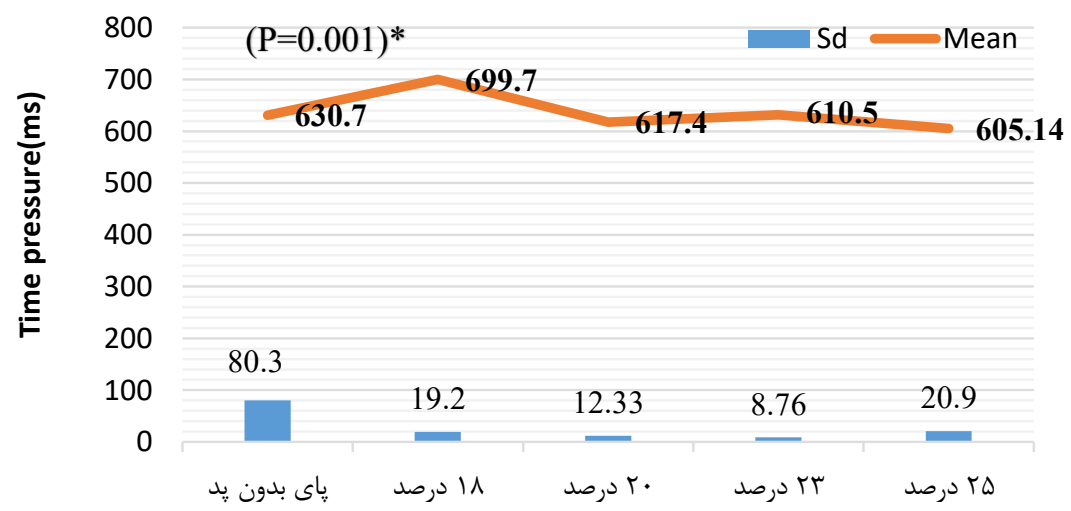

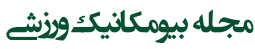

تصوير 9.

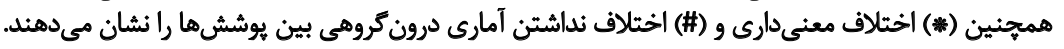

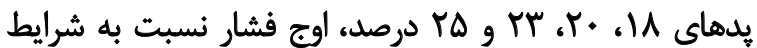

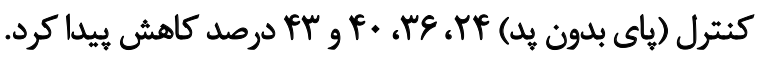

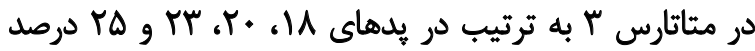

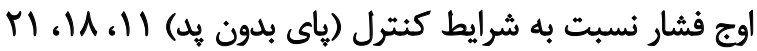

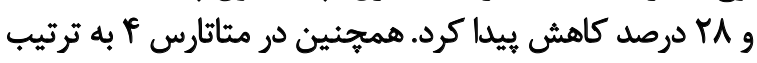

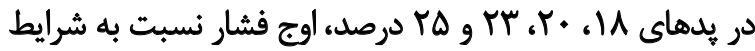

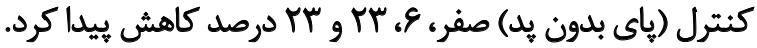

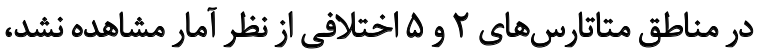

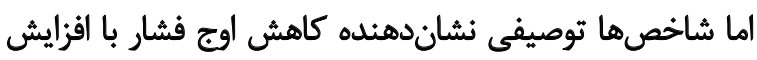
طول يد در اين مناطق بودند.

در يارامتر دوم (اوج زمان اعمال فشار)، اختلاف بين هيوششها

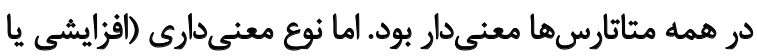

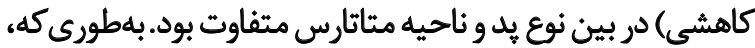

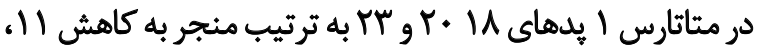

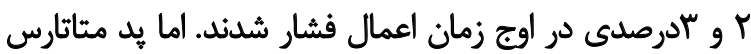

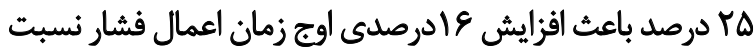

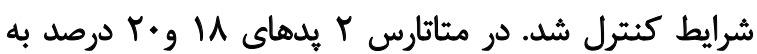

تحليل آمارى اختلاف بين يوششها معنى دار بود (1 + P=1P).

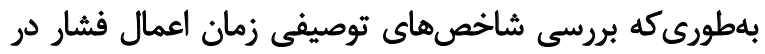

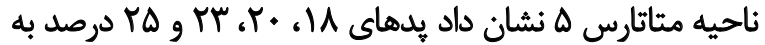

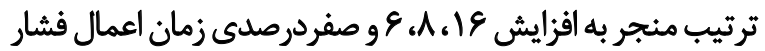

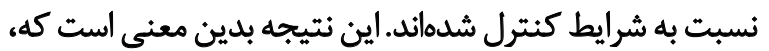

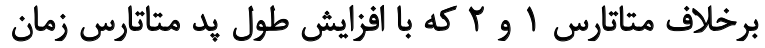

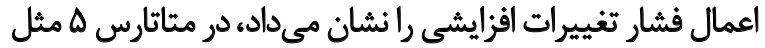

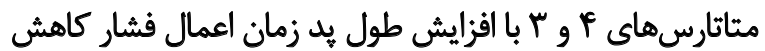

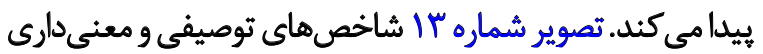

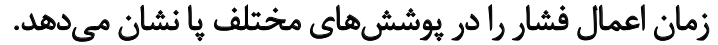

ب

هدف از برُوهش حاضر، بررسي اثر طول مناسب بد متاتارس

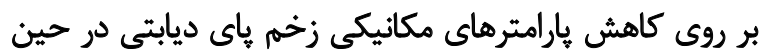

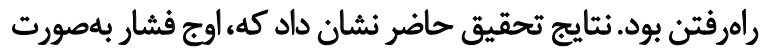

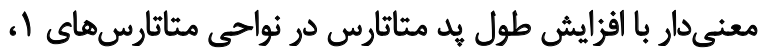

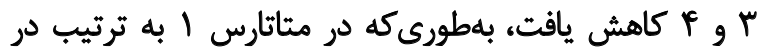

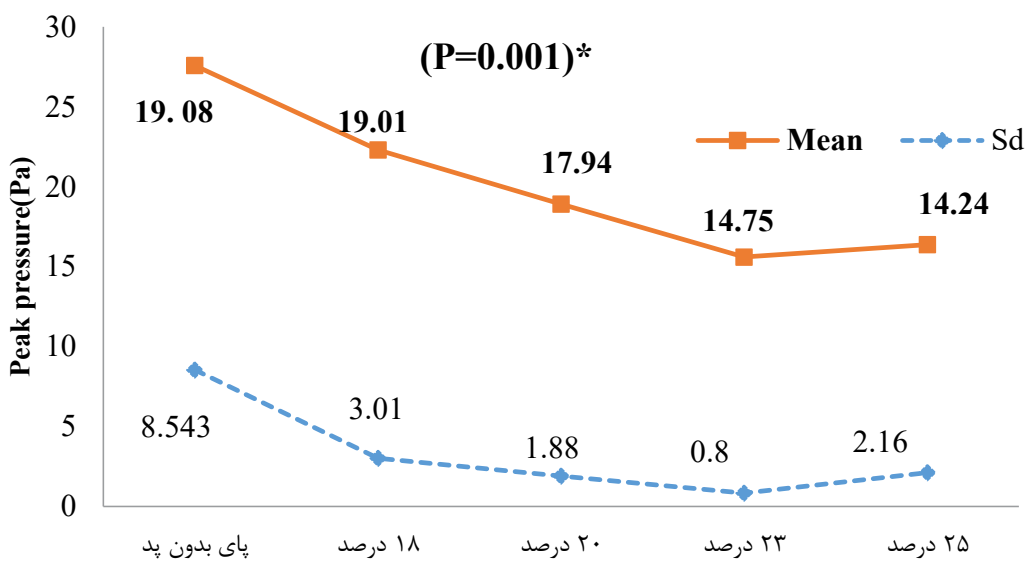

مجله بيومكانيك وزنش

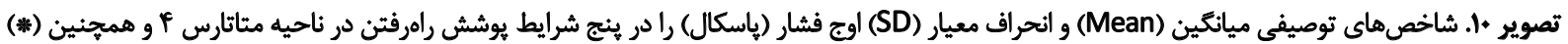

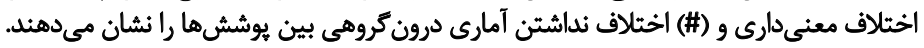




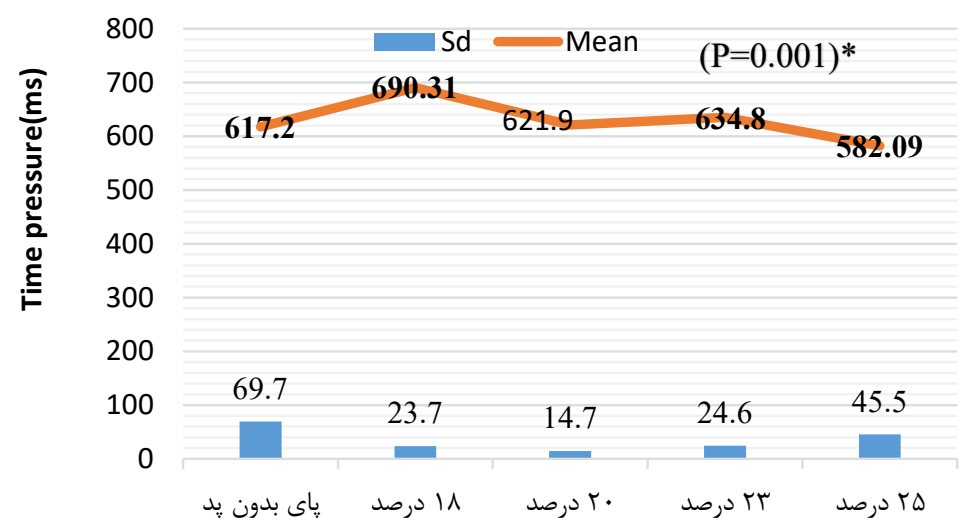

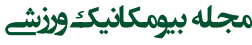

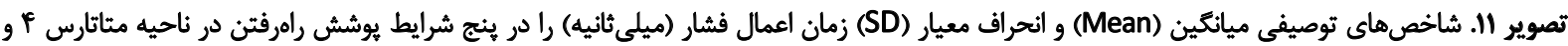

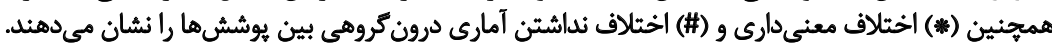

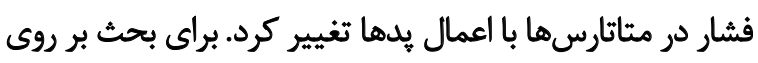

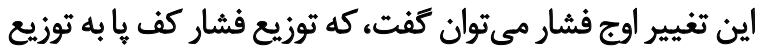

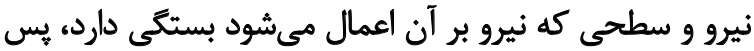

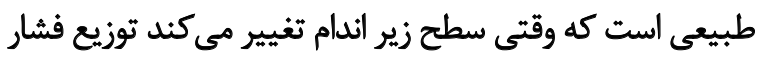

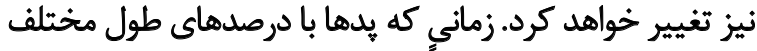

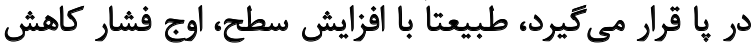

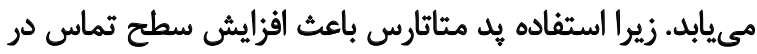

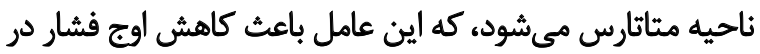

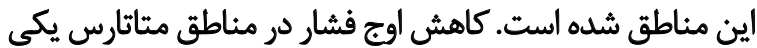
الز مزيتهاي مهمر استفاده بـ است.

اوج زمان اعمال فشار دومين متغير مهم در ايجاد زخم باييى

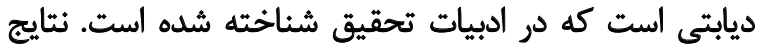

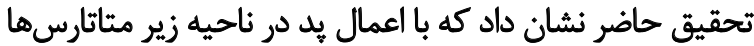

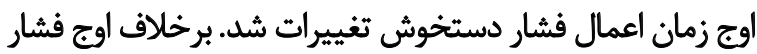

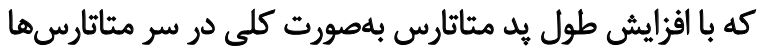

ترتيب منجر به كاهش ب و الادرصدى اوج زمان اعمال فشار

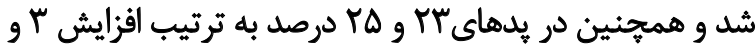

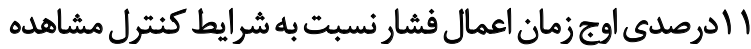

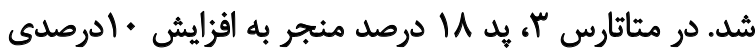

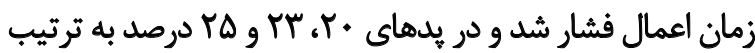

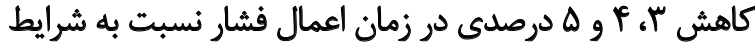

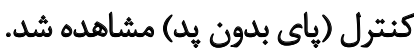

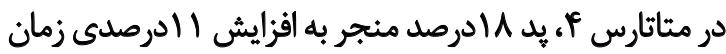

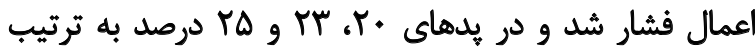

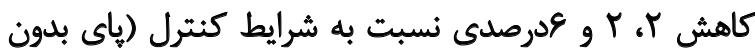

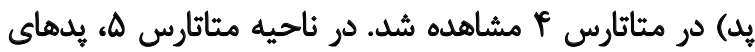

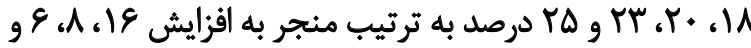

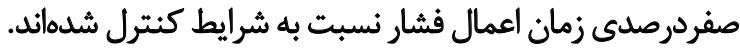
با توجه به نتايج تحقيق، الكوى توزيع فشار كف باوزمان اعمال

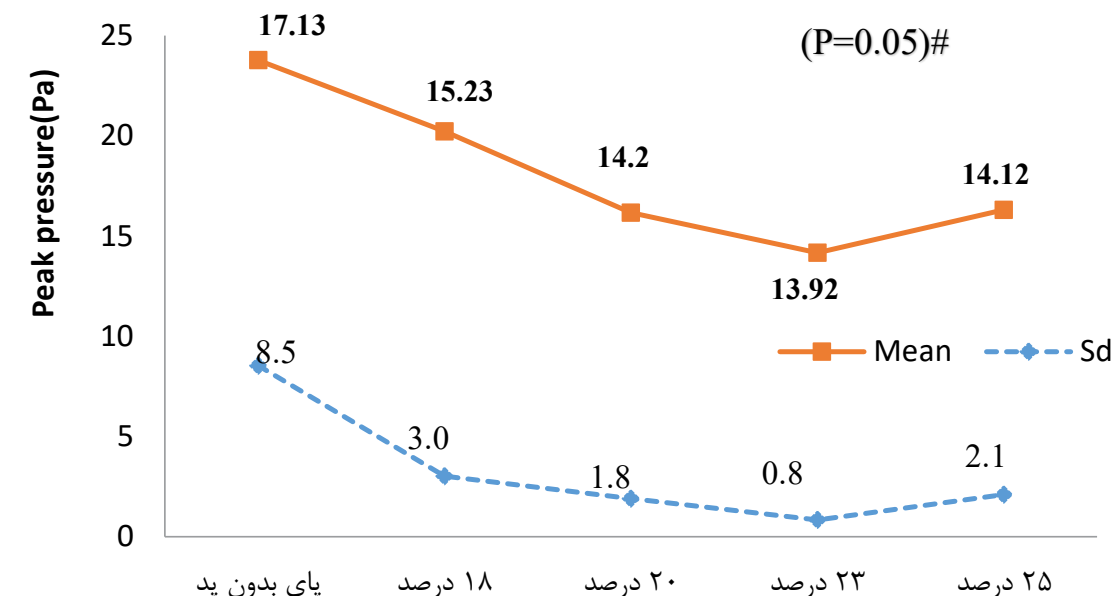

مجله بيومكانيك ورنش

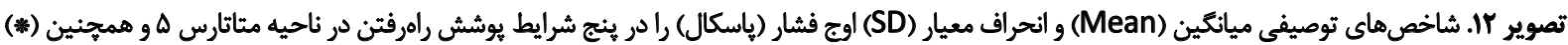

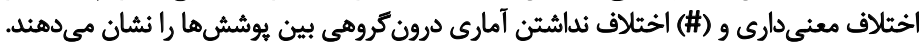




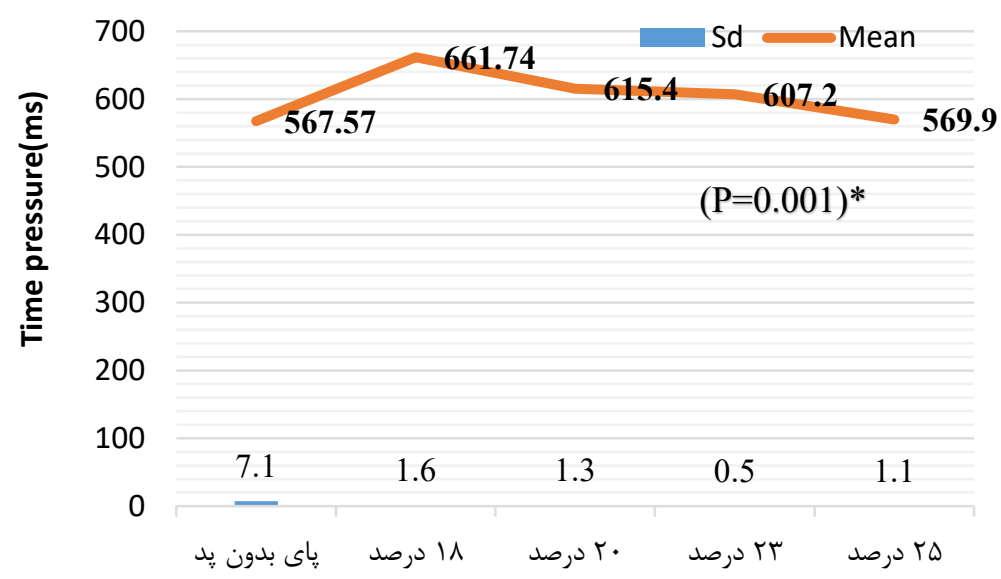

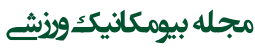

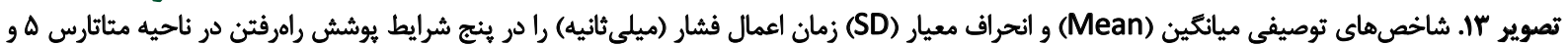

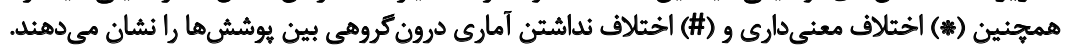

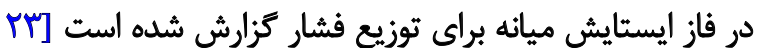
(IV.

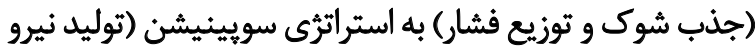

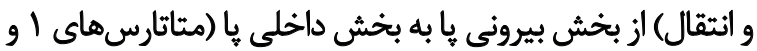

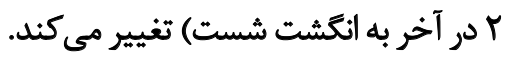

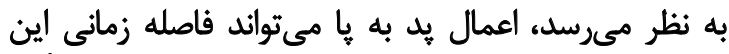

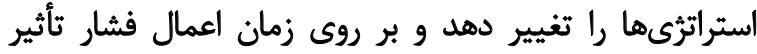

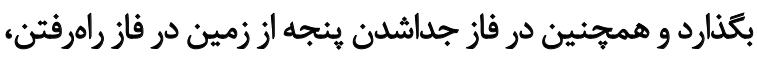
استراتثى مفصلى يا بر روى توليد نيرو است و بايستى مفئ مفاصل

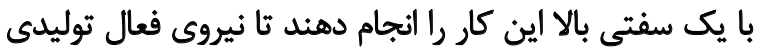

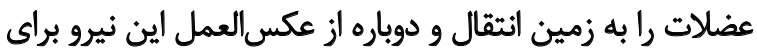

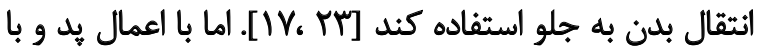

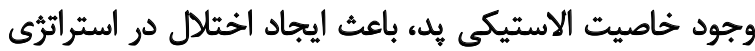

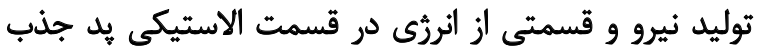

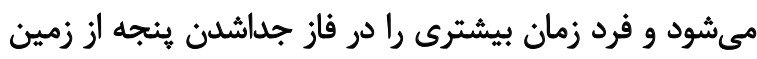

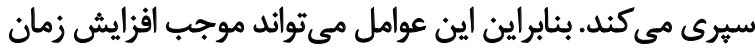

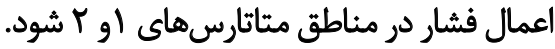

با توجه به نتايج مطالعات، زمان اعمال فشار در مناطق ئنجه

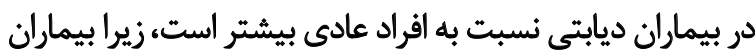

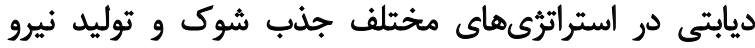

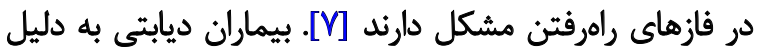

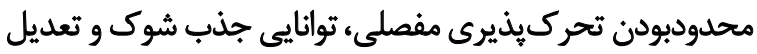

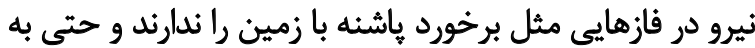

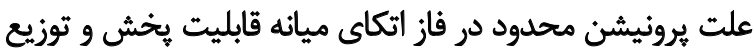

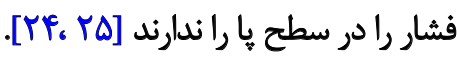

از طرفي در بيماران ديابتي خصوصاً آنهايي كه داراى اختّلال

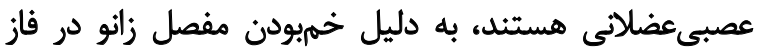

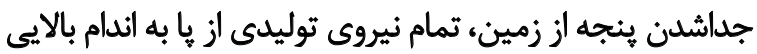

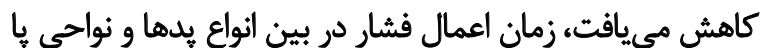

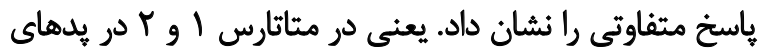

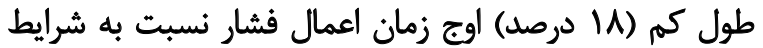

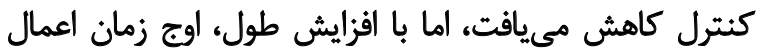

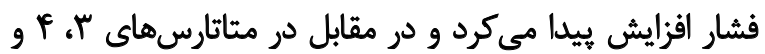

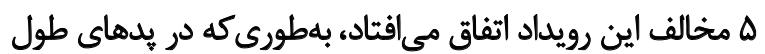

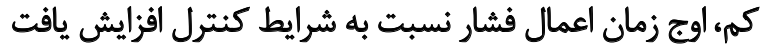

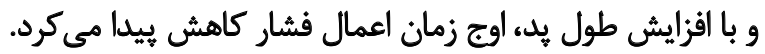

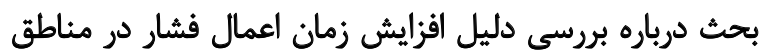

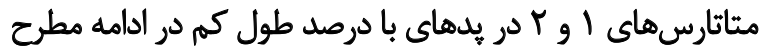
خواهد شد.

به نظر مىرسد افزايش زمان اعمال فشار ارتباطى با تغائير

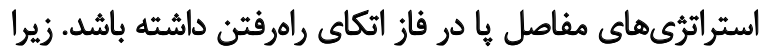

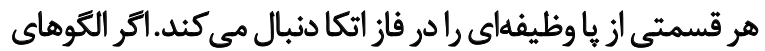

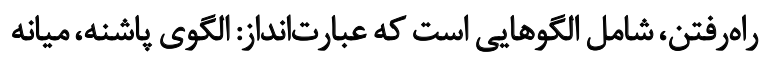

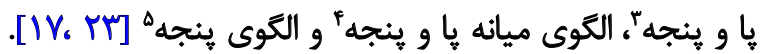

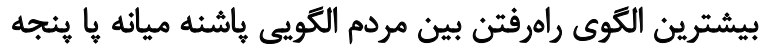

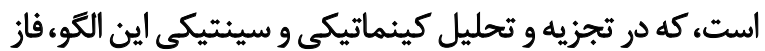

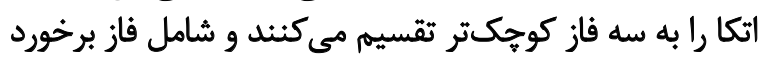

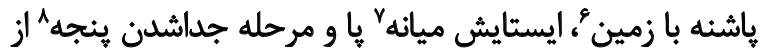

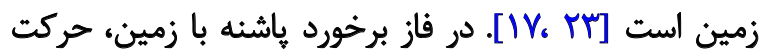

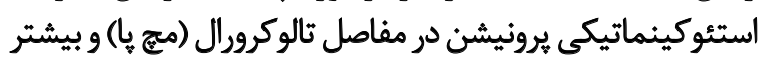
ساب تالار براى جذب شوك و تعديل نيرو است كه اوج بروني برونيشن

3. Heel contact, mid food \& toe off

4. Mid food \& toe off

5. Toe

6. Heel contact

7. Mid stance

8. Toe off 


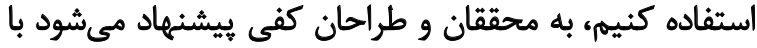

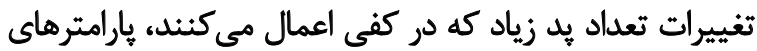

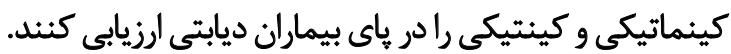

\section{نتيجليَيرىنهايى}

در اين ئروهش مشاهده شده كه بيشترين تأثير يد مثاتارس

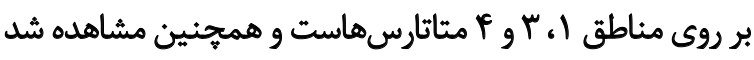

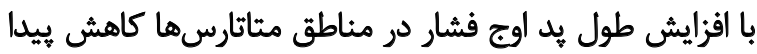

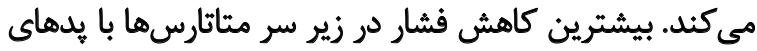

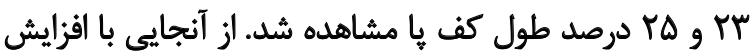

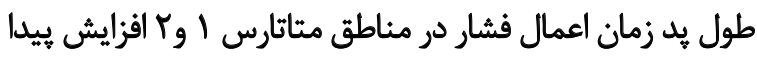

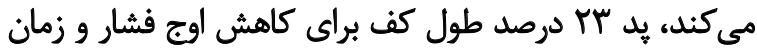

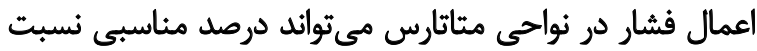

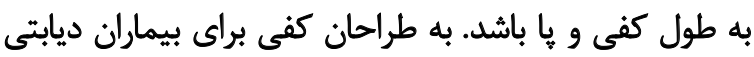

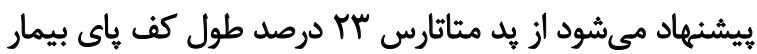
براى طراحى كفى استفاده كنيند.

\section{مالاحظات اخلاقى}

\section{ييروى از اصول اخلاق يُوهش}

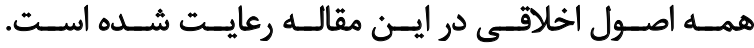

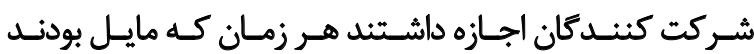

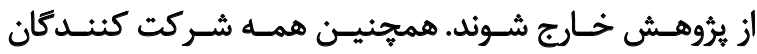

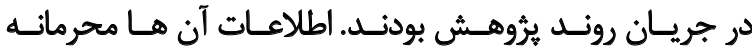

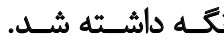

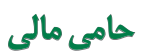

اين مقاله حامى مالى ندارد.

مشاركت نويسند مكَان

تمـام نويســندكان در آمادهســازى ايــن مقالـهـ مشــاركت داشـــتهاند.

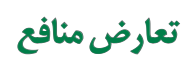

بنابر اظهار نويسندكان اين مقاله تعارض منافع ندارد.

$$
\text { تشكر و قدردانى }
$$

در يايان مقاله لازم ميدانيم از همكارى كليه مسئولين

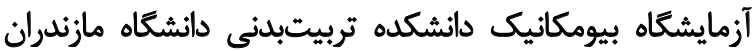

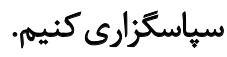

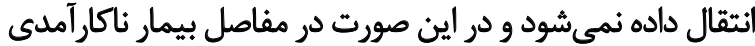

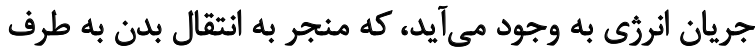

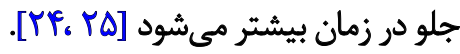

به علت محدودبودن مفصل متاتارس و فلنرياى اول بعضى إنى إني

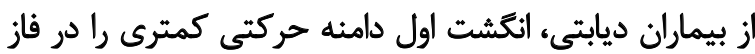

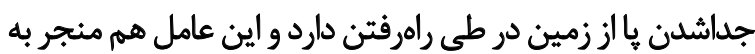

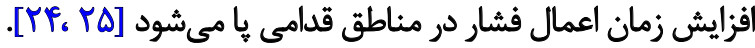

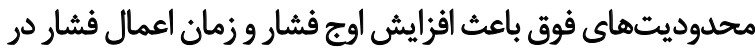

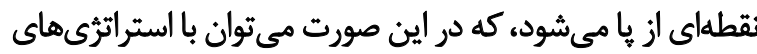

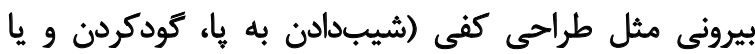

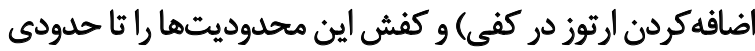

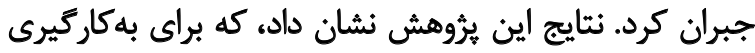

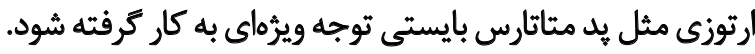

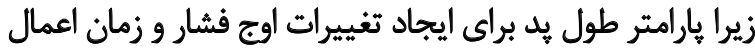

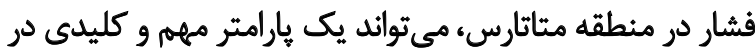

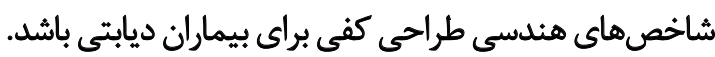
بر اساس تحقيق برخى از محققان مثل هايدا و همكاران،

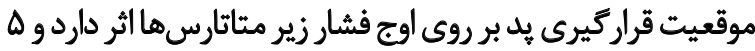

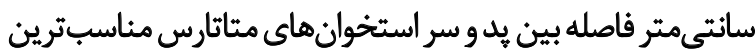

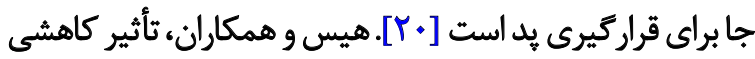

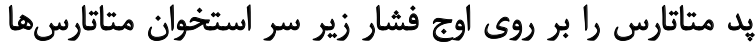

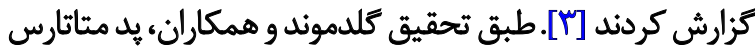

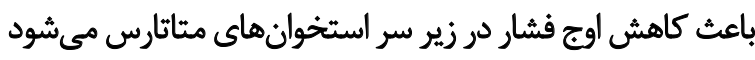

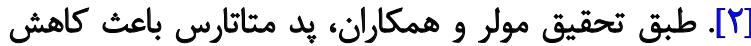

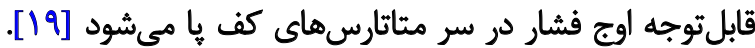

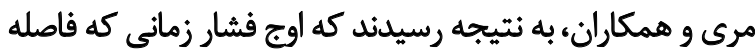

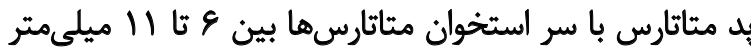

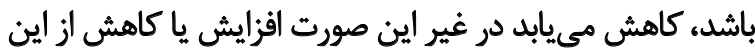

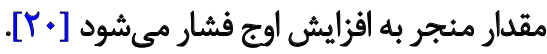

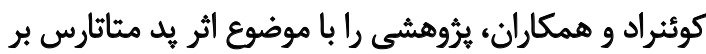

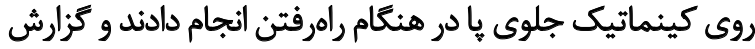

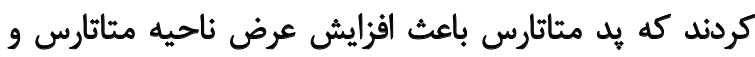

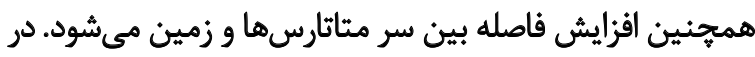

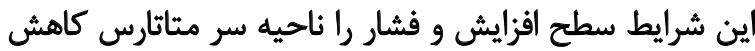

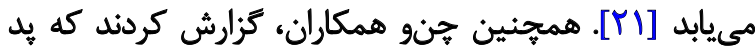

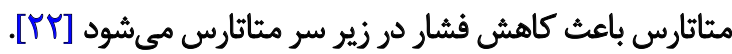

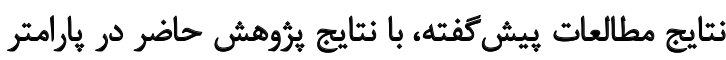

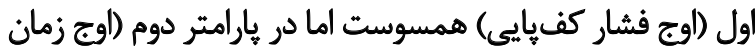

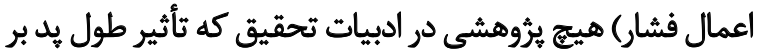

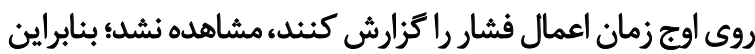

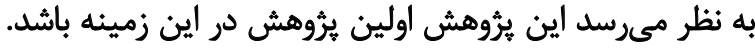

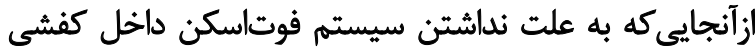

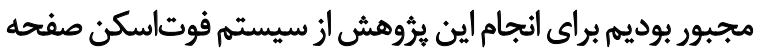




\section{References}

[1] Larijani B, Hasani Ranjbar S. Overview of diabetic foot; novel treatments in diabetic foot ulcer. DARU Journal of Pharmaceutical Sciences. 2008; 16(Suppl. 1):1-6.

[2] Guldemond NA, Leffers P, Schaper NC, Sanders AP, Nieman F, Willems $P$, et al. The effects of insole configurations on forefoot plantar pressure and walking convenience in diabetic patients with neuropathic feet. Clinical Biomechanics. 2007; 22(1):81-7. [DOI:10.1016/j.clinbiomech.2006.08.004] [PMID]

[3] van Schie CH, Rawat F, Boulton AJ. Reduction of plantar pressure using a prototype pressure-relieving dressing. Diabetes Care. 2005; 28(9):22367. [DOI:10.2337/diacare.28.9.2236] [PMID]

[4] Rogers LC, Lavery LA, Armstrong DG. The right to bear legs-an amendment to healthcare: How preventing amputations can save billions for the US health-care system. Journal of the American Podiatric Medical Association. 2008; 98(2):166-8. [DOI:10.7547/0980166] [PMID]

[5] Ledoux WR, Hillstrom HJ. The distributed plantar vertical force of neutrally aligned and pes planus feet. Gait \& Posture. 2002; 15(1):1-9. [DOI:10.1016/S0966-6362(01)00165-5]

[6] Yavuz M. Plantar shear stress distributions in diabetic patients with and without neuropathy. Clinical Biomechanics. 2014; 29(2):223-9. [DOI:10.1016/j.clinbiomech.2013.11.003] [PMID] [PMCID]

[7] Ko SU, Stenholm S, Chia CW, Simonsick EM, Ferrucci L. Gait pattern alterations in older adults associated with type 2 diabetes in the absence of peripheral neuropathy-results from the Baltimore Longitudinal Study of Aging. Gait \& Posture. 2011; 34(4):548-52. [DOI:10.1016/j.gaitpost.2011.07.014] [PMID] [PMCID]

[8] Cavanagh PR. Therapeutic footwear for people with diabetes. Diabetes/ Metabolism Research and Reviews. 2004; 20(S1):S51-S5. [DOI:10.1002/ dmrr.435] [PMID]

[9] Landorf KB, Keenan AM. Efficacy of foot orthoses; What does the literature tell us? Journal of the American Podiatric Medical Association 2000; 90(3):149-58. [DOI:10.7547/87507315-90-3-149] [PMID]

[10] Bus SA, Ulbrecht JS, Cavanagh PR. Pressure relief and load redistribution by custom-made insoles in diabetic patients with neuropathy and foot deformity. Clinical Biomechanics. 2004; 19(6):629-38. [DOI:10.1016/j.clinbiomech.2004.02.010] [PMID]

[11] Milani TL, Schnabel G, Hennig EM. Rear foot motion and pressure distribution patterns during running in shoes with Varus and valgus wedges. Journal of applied biomechanics. 1995; 11(2):177-87. [DOI:10.1123/ jab.11.2.177]

[12] Woodburn J, Helliwell PS. Relation between heel position and the distribution of forefoot plantar pressures and skin callosities in rheumatoid arthritis. Annals of the Rheumatic Diseases. 1996; 55(11):806-10. [DOI:10.1136/ard.55.11.806] [PMID] [PMCID]

[13] Van Gheluwe B, Dananberg HJ. Changes in plantar foot pressure with in-shoe Varus or valgus wedging. Journal of the American Podiatric Medical Association. 2004; 94(1):1-11. [DOI:10.7547/87507315-94-1-1] [PMID]

[14] Kakihana W, Akai M, Yamasaki N, Takashima T, Nakazawa K. Changes of joint moments in the gait of normal subjects wearing laterally wedged insoles. American Journal of Physical Medicine \& Rehabilitation. 2004 83(4):273-8. [DOI:10.1097/01.PHM.0000118035.71195.DE] [PMID]

[15] Meng ZL, Yuan WX, Kang YS. Plantar pressure distribution during barefoot and shod race walking. Journal of Biomechanics. 2007; 40(2):S534. [DOI:10.1016/S0021-9290(07)70524-9]
[16] Kavros SJ, Van Straaten MG, Wood KA, Kaufman KR. Forefoot plantar pressure reduction of off-the-shelf rocker bottom provisional footwear Clinical Biomechanics. 2011; 26(7):778-82. [DOI:10.1016/j.clinbiomech.2011.03.009] [PMID]

[17] Oatis CA. Kinesiology: The mechanics \& pathomechanics of human movement, 2004. Philidelphia: Lippincott Williams \& Wilkins; 2004.

[18] Elftman N. Clinical management of the neuropathic limb. Journal of Prosthetics and Orthotics. 1992; 4(1):1-12. [DOI:10.1097/00008526 199100410-00001

[19] Mueller MJ, Lott DJ, Hastings MK, Commean PK, Smith KE, Pilgram TK. Efficacy and mechanism of orthotic devices to unload metatarsal heads in people with diabetes and a history of plantar ulcers. Physical Therapy. 2006; 86(6):833-42. [PMID]

[20] Hastings MK, Mueller MJ, Pilgram TK, Lott DJ, Commean PK, Johnson JE. Effect of metatarsal pad placement on plantar pressure in people with diabetes mellitus and peripheral neuropathy. Foot \& Ankle International. 2007; 28(1):84-8. [DOI:10.3113/FAI.2007.0015] [PMID]

[21] Koenraadt KL, Stolwijk NM, van den Wildenberg D, Duysens J, Keijsers NL. Effect of a metatarsal pad on the forefoot during gait. Journal of the American Podiatric Medical Association. 2012; 102(1):18-24 [DOI:10.7547/1020018] [PMID]

[22] Chen WM, Lee SJ, Lee PV. Plantar pressure relief under the metatarsal heads-Therapeutic insole design using three-dimensional finite element model of the foot. Journal of Biomechanics. 2015; 48(4):659-65. [DOI:10.1016/j.jbiomech.2014.12.043] [PMID]

[23] Mow VC, Huiskes R. Basic orthopaedic biomechanics \& mechano-biology. Philadelphia: Lippincott Williams \& Wilkins; 2005.

[24] Walker SC, Helm PA, Lavery LA. Gait pattern alteration by functional sensory substitution in healthy subjects and in diabetic subjects with peripheral neuropathy. Archives of Physical Medicine and Rehabilitation. 1997; 78(8):853-6. [DOI:10.1016/S0003-9993(97)90199-4]

[25] Frykberg RG, Zgonis T, Armstrong DG, Driver VR, Giurini JM, Kravitz SR, et al. Diabetic foot disorders: A clinical practice guideline (2006 revision). The Journal of Foot and Ankle Surgery. 2006; 45(5):S1-S66. [DOI:10.1016/S1067-2516(07)60001-5] 